\title{
CHVP Regimen
}

National Cancer Institute

\section{Source}

National Cancer Institute. CHVP Regimen. NCI Thesaurus. Code C9551.

A chemotherapy regimen consisting of cyclophosphamide, doxorubicin, prednisone and teniposide that may be used in the treatment of follicular lymphoma. 